\title{
Knowledge Management Systems for Supporting Enterprise Wide Optimization and Modeling: Strategic and Tactical decisions
}

\author{
Edrisi Muñoz Mata ${ }^{1,2}$, Elisabet Capon García ${ }^{2}$, Jose M. Lainez Aguirre ${ }^{3}$, Konrad \\ Hungerbuehle $^{2}$, Antonio Espuña Camarasa ${ }^{4}$, Luis Puigjaner Corbella ${ }^{4}$ \\ ${ }^{1}$ Centro de Investigacion en Matematicas, Guanajuato, \\ Mexico \\ ${ }^{2}$ ETH Zürich, Department of Chemistry and Applied Biosciences, Zürich, \\ Switzerland \\ ${ }^{3}$ University at Buffalo, Department of Industrial and Systems Engineering, Amherst, NY, \\ USA \\ ${ }^{4}$ Universitat Politecnica de Catalunya, Department of Chemical Engineering, Barcelona, \\ Spain
}

emunoz@cimat.mx, \{elisabet.capon, konrad.hungerbuehler\}@ chem.ethz.ch, jmlainez@gmail.com, \{antonio.espuna, luis.puigjaner\}@upc.edu

\begin{abstract}
Industries constitute a highly complex process network including multiple business and process units, which interact with each other. Enterprisewide optimization addresses the optimization of supply operations, manufacturing and distribution in process industries. This work presents the evolution of knowledge management approaches for supporting enterprise wide optimization in process system engineering.
\end{abstract}

Keywords: Knowledge management systems, enterprise wide optimization.

\section{Introduction}

The chemical process industry is the base for producing a multitude of goods worldwide. Industries constitute a highly complex process network including multiple business and process units, which interact with each other. In order to tackle the aforementioned complexity, collaboration among different scientific disciplines, namely chemical engineering, operations research and computer science is of utmost importance. It is important to underscore that process system engineering (PSE) is a well-established discipline of chemical engineering which covers a set of methods and tools to support decision-making for the creation and operation of the process supply chain constituting the discovery, design, manufacturing and distribution of chemical products from a holistic approach. In this sense, the so-called enterprise-wide optimization (EWO) addresses the optimization of supply operations, manufacturing and distribution in process industries [1]. Specifically, EWO aims to develop flexible modeling environments for the chemical supply chain representation, which is the main 
basis for reaching efficient decision-making. An important challenge in this arena is the integration and management of data, information and decisions along the various hierarchical levels of the company and the whole supply chain. Recent efforts have been devoted to reach a better transactional systems integration in order to improve the use of data for analytical models.

This work presents the evolution of knowledge management approaches for supporting EWO in PSE. In order to deal with the problem complexity, the proposed approaches decouple the system across a hierarchy of appropriately chosen levels. Here, we will focus on the strategic and tactical levels. The knowledge management solution that we proposed has been continuously broadening its scope. We will chronologically summarize such extension. The knowledge management systems was originally created to deal with batch process, however its functionality was later extended to include supply chain planning, life cycle assessment, mathematical model and operation research modeling.

\section{Decision-Making and Modeling at the Chemical Process Industry}

For years, companies have been developing information systems to help people exploiting data, models and information with the purpose of supporting decisionmaking. Nowadays, global competition has made decisions related to certain manufacturing characteristics such economic efficiency, product quality, flexibility, and reliability essential for the viability of the enterprise [2].

Decision support systems (DSS) are information technology solutions that can be used to support complex decision-making. DSS are defined as "aid computer systems at the management company level that combine data and sophisticated analytic models to support decision-making" [3]. A Classic DSS comprises different components, which interact along functionalities at different levels of development and implementation. The first functionality relates to sophisticated database management capabilities with access to internal and external data, information, and knowledge. The next functionality concerns to the modeling functions accessed by a model management system. Other functionality regards to user interfaces design that enable interactive queries, reporting, and graphing functions. Finally, the optimization functionality is found which is supported by mathematic algorithms and/or intuition/knowledge [4].

One of the major challenges of DDS concerns to functionality modeling, which attempt of devising an approximate representation of a system with the goal of providing predictions of its performance. Such a representation is called a model. A model formalizes the relationship between various flows of information and can adopt different forms which range from spreadsheets to complex mathematical programs, neural networks, and expert systems among others. Furthermore, a model is designed to capture certain behavioral aspects of the modeled system - those that are of interest to the analyst - in order to gain knowledge and insight into the system's behavior [5].

A general taxonomy for modeling systems distinguishes between transactional and analytical modeling approaches. Transactional systems are concerned with the acquisition, processing and communication of data over the enterprise. Analytical techniques introduce some reasoning to evaluate the problems, and are further classified 
into descriptive and normative models. Descriptive models can be used to analyze a system, but not to improve it, and provide a better understanding of internal and external functional relationships in the enterprise. Forecasting models, cost relationships, resource utilization relationships, and simulation models fall into this category. On the other hand, optimization or normative models are developed as decision-support systems to assist managers in the identification of efficient and improved decisions.

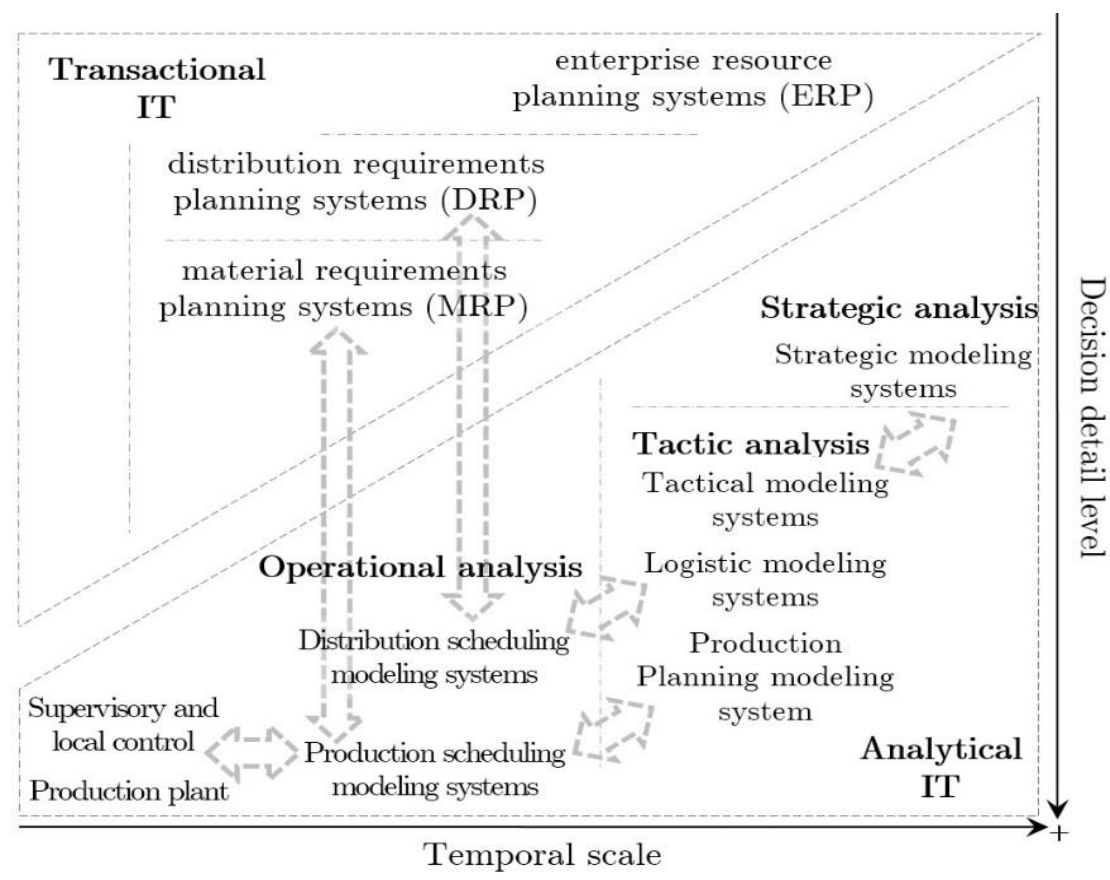

Fig. 1. Hierarchical scheme of modeling systems within organization.

In general, descriptive and optimization algorithms can be broadly classified into equation-oriented or procedure-oriented approaches. Equation-oriented approaches involve rigorous mathematical programs, either deterministic or stochastic, constraint programming and graph theory. Procedure-oriented approaches comprise rule-based techniques, heuristics, and meta-heuristics such as simulated annealing, genetic algorithms (GA), or taboo search. These techniques are based on generic principles and schemes and attempt to improve a given solution time effectively, but the optimality and convergence are difficult to assess. In addition, there is no systematic procedure for obtaining good bounds on the attainable optimum values of the objective function [6].

The basis for solving a systems problem is to formulate a representation in an adequate model that captures the features relevant to efficiently support decisionmaking. Precisely, decision making in process industries results in a highly challenging task. Figure 1 represents a hierarchy of modeling systems that can be found in process system engineering. They are organized based on the temporal scale and the level of decision [7].

The need to integrate the different modeling approaches in a hierarchical decisionsupport system makes necessary the use of consistent terminology and concepts to 
improve the communication and collaboration tasks over the entire system. We believe that a knowledge management system is a suitable tool for this task.

\section{Knowledge Management}

Knowledge management is about leveraging corporate knowledge for greater productivity, value, and competitiveness [8]. How to manage this knowledge has become an important issue in the past few decades, and the knowledge management community has developed a wide range of technologies and applications for both academic research and practical applications. Recent technological advances have made knowledge management expand in a rapidly manner. Enterprises are not apart from this trend.

Along this line, ontology is the knowledge integration of different representations of knowledge at different levels of formalization. The experts who participate in the ontology process are allowed to use their own terminology, facilitating knowledge integration with cooperative tools [9]. Ontologies are increasingly seen as a key semantic technology for addressing heterogeneities and mitigating the problems they create and for enabling semantics-driven knowledge processing. Ontologies are formal structures enabling acquiring, maintaining, accessing, sharing and reusing information [10]. Knowledge management systems benefit from ontologies that semantically enrich information and precisely define the meaning of various information artifacts.

From the essence of enterprise modeling, we could say that ontologies play a critical role in this integration, enabling better analysis of their performance, and management of their operations. An enterprise model is a computational representation of the structure, activities, processes, information, resources, people, behavior, goals, and constraints of a business, government, or other enterprise. The role of an enterprise model is to achieve model-driven enterprise design, analysis, and operation $[11,12]$. In summary, ontologies provide a shared and common understanding of a domain, and an explicit conceptualization that describes the semantics of the enterprise's data.

\section{Knowledge management approaches}

This section will present in a brief manner four knowledge approaches developed to improve knowledge management in the PSE domain. These knowledge systems address areas that range from batch process modeling to supply chain domain models. As the reader will notice, different enterprise functions have been covered in order to improve the effectiveness in the decision-making process. In the next sections, we briefly describe the batch process ontology, enterprise ontology project, ontological math representation and operations research ontology in the chronological order of development.

\subsection{Batch Process Ontology (BaPrOn)}

A crucial step for batch process improvement and optimization is to develop information structures which (i) streamline data gathering, and even more, (ii) are 
capable of integrating transactional systems with the analytical tools used in an enterprise. The batch process ontology (BaPrOn) [13] is a generic ontology following and ANS/ISA88 standards $[14,15,16,17]$ which allows creating an infrastructure that should be general enough to be applied to any batch system. Additionally, this ontology may be used as a straightforward guideline for standardizing batch process management and control.

$\mathrm{BaPrOn}$ integrates different concepts regarding batch processes which are categorized, and the relationships between them are examined. As aforementioned, these concepts are structured in accordance with the ANS/ISA-88 standards. Furthermore, this ontology is also aiming at system integration, improving accessing to information quality by the knowledge description, and thus allowed an improved decision-making process. This was possible using common terminology and manufacturing models which simplifies communication between customers and suppliers in the system.

\subsection{Enterprise Ontology Project (EOP)}

Enterprises comprise several functions, such as production, marketing, sales, human resources, logistics, safety and environment, which interact with each other. Specifically, environmental management is closely related to several levels in the enterprise structure, since they share a large amount of data and information and has been a topic that has received attention in the last years for a firm to operate sustainably. Enterprise ontology project (EOP) [18] has been developed as the technology for information and knowledge models sharing for the environmental assessment of the enterprise. The model provides an enterprise decision-making supporting tool by combining different information systems, which adapts and recognizes the different elements associated with the enterprise functions, and facilitates assessing the environmental performance of enterprises. Besides, this semantic model considers the environmental system representation within the various SC decision levels. It shows how the link between transactional and analytical systems and semantic and quantitative models can be exploited to propose environmentally and economically sustainable solutions for the design and operation of SCs. As a result, more environmentally conscious supply chains and production processes can be automatically proposed to support the decision-making.

\subsection{Ontological Math Representation (OMR)}

The basis of decision-making in the enterprise consists in formally representing the system and its sub-systems in models which adequately capture those features which are necessary to reach consistent decisions. Ontological math representation (OMR) [19] aims to semantically representing mathematical models in engineering domain. The ontology encompasses: (i) the mathematical entities themselves and their behavior (i.e. decisions, parameters, constraints, indicators); (ii) the relation-ships among these entities to build mathematical models; and (iii) the properties which allow to relate the mathematical entities to the semantic abstraction of the elements that they represent. Therefore, the entities of the mathematical models can be directly associated with the engineering concepts, which are unified/standardized into the classes, properties, and 
axioms of an existing ontology in the enterprise and process domain. As a result, the links between the mathematical elements and the abstraction of the reality are explicitly formalized in the ontological framework. Therefore, although the modeler is still responsible for establishing such links, this framework provides a tool for formalizing them, thus clarifying and unveiling the assumptions in the modeling process and the relations of the mathematical model to the real system. This approach is advantageous because it provides the possibility to integrate mathematical models from different decision levels into a single platform, and to easily incorporate other system aspects, such as the identification of mathematical elements (e.g. variables, performance indicators) that refer to the same engineering entity.

\subsection{Operations Research Ontology (ORO)}

Finally, we would like to mention an operations research ontology (ORO) semantic model that has been developed as a step forward in capturing the nature of problems and technologies for decision making in the enterprise. Specifically, the whole process of decision making and the creation and classification of equations according to their structure are qualitatively represented in terms of OR principles. This OR ontology is integrated with other two semantic models previously developed, (EOP) and (OMR), thus enhancing the functionalities of the original ontological framework. The scope of these models comprises the representation of the real system for EOP, the mathematical representation domain for OMR, and finally the problem design representation for ORO.

\section{Conclusions}

The ontological frameworks provides a powerful tool to support computational optimization models for strategical and tactic decision-making, allowing the comprehensive application of enterprise wide optimization throughout the process industry. This work contributes to improve communication within plant process environment, and represents a step forward to support the integration of analytical software tools, resulting into an enhancement of the supply chain functions.

Thus, ontological models enhances the way for achieving a successful decisionmaking supporting tool which adapts and recognizes the different elements found recipe models. Moreover, a general semantic framework is proposed, which is able to model any plant layout, proving its re-usability. As a whole, the main contributions of this environment and the model behind are re-usability, usability, higher efficiency in communication and coordination procedures. In addition, it has been proved the adequacy of an ontology as a means for sharing information about a general model for different problem representations. As a result, it solves the problem of integration, standardization and compatibility of heterogeneous modeling systems. Even more, the response time for decision making task could be reduced and better decisions adopted owing to faster availability of higher quality data and the improved visibility of the existing relationships between the scheduling function and other hierarchical levels functions. 
Acknowledgements. Authors would like to acknowledge the Spanish Ministerio de Economía y Competitividad and the European Regional Development Fund for supporting the present research by projects EHMAN (DPI2009-09386) and SIGERA (DPI2012-37154-C02-01). Finally, the financial support received from CIMAT México is also fully acknowledged.

\section{References}

1. Grossmann, I.: Enterprise-wide optimization: A new frontier in process systems engineering. Aiche Journal, Vol. 51, No. 7, pp. 1846-1857 (2005)

2. Venkatasubramanian, V., Zhao, C., Joglekar, G., Jain, A., Hailemariam, L., Suresh, P., Akkisetty, P., Morris, K., Reklaitis, G.: Ontological informatics infrastructure for pharmaceutical product development and manufacturing. Computer and Chemical Engineering 30 (July), pp. 1482-1496 (2006)

3. Simon, F., Murray, T.: Decision support systems. Commun. ACM, Vol. 50, No. 3, pp. 39$40(2007)$

4. Shim, J.P., Warkentin, M., Courtney, F.J., Power, J.D., Sharda, R., Carlsson, C.: Past, present, and future of decision support technology. Decision Support Systems, Vol. 33, No. 2, pp. 111-126 (2002)

5. Morris, W.T.: On the arts of modelling. Management Science, Vol. 13, pp. 707-717 (1967)

6. Pekny, J.F., Reklaitis, G.V.: Towards the Convergence of Theory and Practice: A Technology Guide for Scheduling/Planning Methodology. Foundations of Computer Aided Process Operations (1998)

7. Harjunkoski, I., Nystrom, R., Horch, A.: Integration of scheduling and control-Theory or practice? Computers \& Chemical Engineering, Vol. 33, No. 12, pp. 1909-1918, Pergamon (2009)

8. Schreiber, G., Akkermans, H., Anjewierden, A., de Hoog, R., Shadbolt, N., Van de Velde, W., Wielinga, B.: Knowledge Engineering and Management: The Common KADS Methodology. Cambridge, MA: MIT Press (2000)

9. Fernandez-Breis, J.T. Martinez-Bejar, R.: A cooperative tool for facilitating knowledge management. Expert Systems with Applications, Vol. 18, No. 4, pp. 315-330 (2000)

10. Gruber, T.R.: A translation approach to portable ontology specifications. Knowledge Acquisition, Vol. 5, No. 2, pp. 199-220 (1993)

11. Fox, M.S., Barbuceanu, M., Gruninger, M., Lin, J.: An organization ontology for enterprise modelling. In Modeling, In: International Conference on Enterprise Integration Model-ling Technology 97, Springer (1997)

12. Fox, M.S., Gruninger, M.: Enterprise modeling. AI Magazine, Vol. 19, No. 3, pp. 109-121 (1998)

13. Muñoz, E., Espuña, A., Puigjaner, L.: Towards an ontological infrastructure for chemical batch process management. Comput. Chem. Eng., Vol. 34, pp. 668-682 (2010)

14. International Society for Measurement and Control (1995). Batch control part 1 models and terminology.

15. International Society for Measurement and Control. Control batch parte 4 registros de producción de lote (2006)

16. International Society for Measurement and Control. Batch control part 5 automated equipment control models \& terminology (2007)

17. International Society for Measurement and Control. Isa-88/95 technical report: Using isa88 and isa-95 together. Technical report, ISA The Instrumentation, Systems, and Automation Society (2007) 
Edrisi Muñoz Mata, Elisabet Capon García, Jose M. Lainez Aguirre, Konrad Hungerbuehle, et al.

18. Muñoz, E., Capón-García, E., Lainez, J.M., Espuña, A., Puigjaner, L.: Considering environmental assessment in an ontological framework for enterprise sustainability. Journal of Cleaner Production, Vol. 47, pp. 149-164 (2013)

19. Muñoz, E., Capón-García, E., Lainez, J.M., Espuña, A., Puigjaner, L.: Using mathematical knowledge management to support integrated decision-making in the enterprise. Computers and Chemical Engineering. Vol.66, pp.139-150 (2014) 